\title{
New Method of Solving the Seepage Model for the Multilayer Composite Reservoir with the Double Porosity
}

\author{
Qiang Wang ${ }^{1, *}$, Shunchu $\mathrm{Li}^{1}$, Mei Luo ${ }^{1}$, Dongdong Gui ${ }^{2}$ \\ ${ }^{1}$ Institute of Applied Mathematics, Xihua University, Chengdu, China \\ ${ }^{2}$ Beijing Dong run ke Petroleum Technology Co,Ltd, Beijing, China \\ *Corresponding author: wangqian1205@foxmail.com
}

Received March 19, 2015; Revised April 04, 2015; Accepted April 14, 2015

\begin{abstract}
Aimed at multilayer composite reservoir with the double porosity, meanwhile considering the influence of well bore storage and skin effect, the seepage model for multilayer composite reservoir with the double porosity which the flow was steady from pore to crack was established in different outer boundary (infinite; closed; constant pressure) conditions; the exact solution of reservoir pressure drop and bottom hole pressure drop were obtained by Laplace transform in the Laplace space; the unified expression of solution was obtained by constructing similar kernel functions in different outer boundary conditions, therefore new method which solving this class of reservoir model is put forward, namely similar construction method. This method plays an important guiding role in exploring seepage law of oil and gas reservoir.
\end{abstract}

Keywords: double porosity, multilayer composite reservoir, similar construction method, similar kernel function, similar structure

Cite This Article: Qiang Wang, Shunchu Li, Mei Luo, and Dongdong Gui, "New Method of Solving the Seepage Model for the Multilayer Composite Reservoir with the Double Porosity." American Journal of Energy Research, vol. 3, no. 1 (2015): 8-12. doi: 10.12691/ajer-3-1-2.

\section{Introduction}

Reservoir with the double porosity is reservoir that has the natural fractures [1], it is a kind of reservoir with the complex medium, which consist of two kinds of pore medium, namely matrix block medium and fracture medium, and most of reservoirs have the characteristics of multilayer. Composite reservoir is reservoir that consist of two reservoir area with the different nature[1]. Therefore multilayer composite reservoir with the double porosity is the combination of multilayer reservoir with the double porosity and composite reservoir.

The multilayer reservoir has been researched by some domestic and foreign scholars. In 1986, Kuchuk [2] built model of the multilayer connecting reservoir, and analytic expression of the model in the Laplace space was obtained by using superposition principle. In 1999, considering the influence of the well bore storage and skin effect, aimed at multilayer reservoir without the cross-flow between layers, Xianzhi $\mathrm{Xu}$ etc [3] provided computing method of the type curve for well test model and fitting method of the layering parameter. In 2002, Shunchu Li etc [4] obtained general solution of pressure distribution by using Laplace transform for multilayer reservoir which well bore storage and skin effect were taken into consideration. In 2009, considering cross-flow between layers, Liehui Zhang [5] built seepage model for the double layer reservoir with the double porosity, and typical curve of flow pressure at well bottom was drawn by using Stehfest numerical inverse and computer programming. In 2011, considering the influence of well bore storage and skin effect, Jingjing Guo etc [6] built model without cross-flow for the multilayer reservoir with the double porosity, analytic solution of bottom hole pressure and production of the layer were obtained in the Laplace space by Laplace transform, and according to method of numerical inverse, the changeable curve of production of the layer was drawn. In 2013, Quanyong Li etc [7] solved exact solution of reservoir pressure and bottom hole pressure by Laplace transform for multilayer reservoir with the double porosity, and unified expression of solution was obtained for different outer boundary conditions. However, so far no one studies the seepage model for the multilayer composite reservoir with the double porosity.

In 2004, Shunchu Li and Minhui Jia [8] put forward an important method which solved a class of differential equation, namely similar construction method, just as any a real number can be expressed by continued fraction. In the next years, many studies[9-19] have been carried out for some boundary value problems of the special differential equation. On the basis of above research, considering the influence of well bore storage and skin effect, this paper firstly built the seepage model for the multilayer composite reservoir with the double porosity. We solved similar structure of reservoir pressure and bottom hole pressure in the Laplace space by Laplace 
transform, and this research will further improve the theory of similar structure which solving the seepage model of complex reservoir, and it is also a kind of supplement for theoretical research of the seepage law in reservoir.

\section{Establishment of Model}

In order to build the model of the multilayer composite reservoir with the double porosity. The basic assumptions are included as follows:

(1)The reservoir with the double porosity is level; equal thickness and isotropy,

(2) $N$ layers are level and equal thickness in multilayer composite reservoir, and inter-layer has not cross-flow. The fluid of every layer is homogeneous and weakly compressible. Formation fluid is single-phase, and fluid obeys Darcy's law.

(3) Ignoring gravity and capillary force.

(4) Constant production is $q$ (the yield of the $j$ layer is $\left.q_{j}, q=\frac{1}{N} \sum_{j=1}^{N} q_{j}\right)$.

(5) A well of radius $r_{w}$ (the well radius of the $j$ layer is $r_{w j}$, and $\left.r_{w}=\frac{1}{N} \sum_{j=1}^{N} r_{w j}\right)$ is opened in the center of reservoir, the boundary radius is $\beta r_{w j}(\beta>1)$ in inner region, the boundary radius is $R_{j}\left(R_{j}>\beta r_{w j}\right)$ in outer region.

(6) Initial pressure of every layer is $p_{o j}(j=1,2, \cdots, N)$.

According to above assumptions, the model of the multilayer composite reservoir with the double porosity is built as follows:

\section{Seepage differential equations}

$$
\begin{gathered}
\frac{\partial^{2} P_{1 j f}}{\partial r^{2}}+\frac{1}{r} \frac{\partial P_{1 j f}}{\partial r}+\lambda_{1 j}\left(P_{1 j m}-P_{1 j f}\right) \\
=\delta_{1 j f} \frac{\partial P_{1 j f}}{\partial t}\left(r_{w j}<r<\beta r_{w j}, \mathrm{t}>0, j=1,2 \cdots N\right) \\
\delta_{1 j m} \frac{\partial P_{1 j m}}{\partial t}+\lambda_{1 j}\left(P_{1 j m}-P_{1 j f}\right) \\
=0\left(r_{w j}<r<\beta r_{w j} ; \mathrm{t}>0 ; \mathrm{j}=1,2, \cdots, \mathrm{N}\right) \\
\frac{\partial^{2} P_{2 j f}}{\partial r^{2}}+\frac{1}{r} \frac{\partial P_{2 j f}}{\partial r}+\lambda_{2 j}\left(P_{2 j m}-P_{2 j f}\right) \\
=\delta_{2 j f} \frac{\partial P_{2 j f}}{\partial t}\left(r>\beta r_{w j}, \mathrm{t}>0, j=1,2 \cdots N\right) \\
\delta_{2 j m} \frac{\partial P_{2 j m}}{\partial t}+\lambda{ }_{2 j}\left(P_{2 j m}-P_{2 j f}\right)=0 \\
\left(r>\beta r_{w j} ; \mathrm{t}>0 ; \mathrm{j}=1,2, \cdots, \mathrm{N}\right)
\end{gathered}
$$

\section{Initial conditions}

$$
P_{1 j f}(r, 0)=P_{1 j m}(r, 0)=P_{2 j f}(r, 0)=P_{2 j m}(r, 0)=0
$$

\section{Internal boundary condition}

$$
\left\{\begin{array}{l}
P_{w}(t)=\left[P_{1 j f}-S_{j} r \frac{\partial P_{1 j f}}{\partial r}\right]_{r=r_{w j}} \\
\left.2 \pi \sum_{j=1}^{N} \gamma_{1 j}\left(r \frac{\partial P_{1 j f}}{\partial r}\right)\right|_{r=r_{w j}}=-B q+C \frac{d P_{w}}{d t}
\end{array}\right.
$$

Interface boundary condition

$$
\left\{\begin{array}{l}
P_{1 j f}\left(\beta r_{w j}, \mathrm{t}\right)=P_{2 j f}\left(\beta r_{w j}, \mathrm{t}\right) \\
\left.\frac{\partial P_{1 j f}}{\partial r}\right|_{r=\beta r_{w j}}=\left.\gamma_{j f} \frac{\partial P_{2 j f}}{\partial r}\right|_{r=\beta r_{w j}}
\end{array}\right.
$$

\section{Outer boundary conditions}

When outer boundary is infinity:

$$
P_{2 j f}(\infty, \mathrm{t})=0
$$

When outer boundary is closed:

$$
\left.\frac{\partial P_{2 j f}}{\partial r}\right|_{r=R_{j}}=0
$$

When outer boundary is constant pressure:

$$
P_{2 j f}\left(\mathrm{R}_{j}, \mathrm{t}\right)=0
$$

Where: $\quad P_{n j i}=p_{o j}-p_{n j i}(n=1,2 ; j=1,2 \cdots, N ; i=f, m)$; $\lambda_{n j}=\frac{\alpha_{n j} k_{n j m}}{k_{n j f}} \quad ; \quad \gamma_{1 j}=\frac{k_{1 j f} h_{1 j}}{\mu_{1 j}} \quad ; \quad \gamma_{j f}=\frac{k_{2 j f} \mu_{1 j}}{k_{1 j f} \mu_{2 j}}$; $\delta_{n j i}=\frac{\phi_{n j i} C_{t_{n j i}} \mu_{n j}}{k_{n j f}}$; the subscript “ $i=f$ " indicates fractured media ; the subscript " $i=m$ " indicates matrix blocks; the subscript " $n=1$ " indicates the inner zone and the subscript " $n=2$ " indicates the outer zone.

\section{Laplace Form of the Model}

Applied to models (1)-(10) by employing the Laplace transform method on dimensionless time $t$, Let:

$$
\begin{aligned}
& \bar{P}_{n j i}(r, z) \\
&= \int_{0}^{\infty} e^{-z t} P_{n j i}(r, t) d t(n=1,2 ; j=1,2, \cdots, N ; i=f, m) ; . \\
& \bar{P}_{w}(z)=\int_{0}^{\infty} e^{-z t} P_{w}(t) d t
\end{aligned}
$$

then the boundary value problem of an ordinary differential equation is obtained in the Laplace space as follows:

$$
\begin{gathered}
\frac{d^{2} \bar{P}_{1 j f}}{d r^{2}}+\frac{1}{r} \frac{d \bar{P}_{1 j f}}{d r}+\lambda_{1 j}\left(\bar{P}_{1 j m}-\bar{P}_{1 j f}\right) \\
=\delta_{1 j f} z \bar{P}_{1 j f}\left(r_{w j}<r<\beta r_{w j}, \mathrm{t}>0, j=1,2 \cdots N\right) \\
\delta_{1 j m} z \bar{P}_{1 j m}+\lambda_{1 j}\left(\bar{P}_{1 j m}-\bar{P}_{1 j f}\right)=0 \\
\left(r_{w j}<r<\beta r_{w j} ; \mathrm{t}>0 ; \mathrm{j}=1,2, \cdots, \mathrm{N}\right)
\end{gathered}
$$




$$
\begin{aligned}
& \frac{d^{2} \bar{P}_{2 j f}}{d r^{2}}+\frac{1}{r} \frac{d \bar{P}_{2 j f}}{d r}+\lambda_{2 j}\left(\bar{P}_{2 j m}-\bar{P}_{2 j f}\right)=\delta_{2 j f} z \bar{P}_{2 j f} \\
& \left(r>\beta r_{w j}, \mathrm{t}>0, j=1,2 \cdots N\right) \\
& \delta_{2 j m} z \bar{P}_{2 j m}+\lambda_{2 j}\left(\bar{P}_{2 j m}-\bar{P}_{2 j f}\right)=0 \\
& \left(r>\beta r_{w j} ; \mathrm{t}>0 ; \mathrm{j}=1,2, \cdots, \mathrm{N}\right) \\
& \left\{\begin{array}{l}
\bar{P}_{w}(z)=\left[\bar{P}_{1 j f}-S_{j} r \frac{d \bar{P}_{1 j f}}{d r}\right]_{r=r_{w j}} \\
\left.2 \pi \sum_{j=1}^{N} \gamma_{1 j}\left(r \frac{d \bar{P}_{1 j f}}{d r}\right)\right|_{r=r_{w j}}=-\frac{B q}{z}+C z \bar{P}_{w}
\end{array}\right. \\
& \left\{\begin{array}{l}
\bar{P}_{1 j f}\left(\beta r_{w j}, \mathrm{z}\right)=\bar{P}_{2 j f}\left(\beta r_{w j}, \mathrm{z}\right) \\
\left.\frac{d \bar{P}_{1 j f}}{d r}\right|_{r=\beta r_{w j}}=\left.\gamma_{j f} \frac{d \bar{P}_{2 j f}}{d r}\right|_{r=\beta r_{w j}}
\end{array}\right. \\
& \bar{P}_{2 j f}(\infty, \mathrm{z})=0 \\
& \left.\frac{d \bar{P}_{2 j f}}{d r}\right|_{r=R_{j}}=0 \\
& \bar{P}_{2 j f}\left(\mathrm{R}_{j}, \mathrm{z}\right)=0
\end{aligned}
$$

\section{Solution of the Model and Similar Structure}

After the Laplace transform, equation(12) and (14) plug into the equation (11) and (13) respectively, and then Bessel equation of 0 order is obtained, therefore the general solutions $^{[20]}$ of equation (11)-(14) are obtained as follows:

$$
\begin{gathered}
\bar{P}_{1 j f}(r, z)=A_{j 1} K_{0}\left[r \sqrt{f_{1 j}(z)}\right]+B_{j 1} I_{0}\left[r \sqrt{f_{1 j}(z)}\right] \text { (18) } \\
\bar{P}_{1 j m}(r, z)=\frac{\lambda_{1 j}}{\delta_{1 j m} z+\lambda_{1 j}}\left\{\begin{array}{l}
A_{j 1} K_{0}\left[r \sqrt{f_{1 j}(z)}\right] \\
+B_{j 1} I_{0}\left[r \sqrt{f_{1 j}(z)}\right]
\end{array}\right\} \quad \text { (19) } \\
\bar{P}_{2 j f}(r, z)=A_{j 2} K_{0}\left[r \sqrt{f_{2 j}(z)}\right]+B_{j 2} I_{0}\left[r \sqrt{f_{2 j}(z)}\right] \text { (20) } \\
\bar{P}_{2 j m}(r, z)=\frac{\lambda_{2 j}}{\delta_{2 j m} z+\lambda_{2 j}}\left\{\begin{array}{l}
A_{j 2} K_{0}\left[r \sqrt{f_{2 j}(z)}\right] \\
+B_{j 2} I_{0}\left[r \sqrt{f_{2 j}(z)}\right]
\end{array}\right\} \quad(21)
\end{gathered}
$$

Where:

$$
f_{n j}(z)=\frac{\delta_{n j f} \delta_{n j m} z^{2}+\delta_{n j f} z \lambda_{n j}+\delta_{n j m} z \lambda_{n j}}{\delta_{n j m} z+\lambda_{n j}}(n=1,2),
$$

$A_{j 1} 、 B_{j 1}, A_{j 2} 、 B_{j 2}$ are undetermined coefficients, and they are determined by internal and outer boundary conditions.

The following equations are obtained by putting the formula (18)-(21) plug into the formula (15)-(17).

$$
\begin{aligned}
& \bar{P}_{w}(z)=\left\{\begin{array}{l}
K_{0}\left[r_{w j} \sqrt{f_{1 j}(z)}\right] \\
+S_{j} r_{w j} \sqrt{f_{1 j}(z)} K_{1}\left[r_{w j} \sqrt{f_{1 j}(z)}\right]
\end{array}\right\} A_{j 1} \\
& +\left\{\begin{array}{l}
I_{0}\left[r_{w j} \sqrt{f_{1 j}(z)}\right] \\
-S_{j} r_{w j} \sqrt{f_{1 j}(z)} I_{1}\left[r_{w j} \sqrt{f_{1 j}(z)}\right]
\end{array}\right\} B_{j 1} \\
& 2 \pi \sum_{j=1}^{N} \gamma_{j 1} r_{w j}\left\{\begin{array}{l}
-\sqrt{f_{1 j}(z)} K_{1}\left(r_{w j} \sqrt{f_{1 j}(z)}\right) A_{j 1} \\
+\sqrt{f_{1 j}(z)} I_{1}\left(r_{w j} \sqrt{f_{1 j}(z)}\right) B_{j 1}
\end{array}\right\} \\
& =-\frac{B q}{z}+C z \bar{P}_{w} \\
& K_{0}\left[\beta r_{w j} \sqrt{f_{1 j}(z)}\right] A_{j 1}+I_{0}\left[\beta r_{w j} \sqrt{f_{1 j}(z)}\right] B_{j 1} \\
& -K_{0}\left[\beta r_{w j} \sqrt{f_{2 j}(z)}\right] A_{j 2}-I_{0}\left[\beta r_{w j} \sqrt{f_{2 j}(z)}\right] B_{j 2}=0 \\
& \sqrt{f_{1 j}(z)} K_{1}\left[\beta r_{w j} \sqrt{f_{1 j}(z)}\right] A_{j 1} \\
& -\sqrt{f_{1 j}(z)} I_{1}\left[\beta r_{w j} \sqrt{f_{1 j}(z)}\right] B_{j 1} \\
& -\gamma_{j f} \sqrt{f_{2 j}(z)} K_{1}\left[\beta r_{w j} \sqrt{f_{2 j}(z)}\right] A_{j 2} \\
& +\gamma_{j f} \sqrt{f_{2 j}(z)} I_{1}\left[\beta r_{w j} \sqrt{f_{2 j}(z)}\right] B_{j 2}=0 \\
& B_{j 2}=0 \\
& \sqrt{f_{2 j}(z)} K_{1}\left[R_{j} \sqrt{f_{2 j}(z)}\right] A_{j 2} \\
& -\sqrt{f_{2 j}(z)} I_{1}\left[R_{j} \sqrt{f_{2 j}(z)}\right] B_{j 2}=0 \\
& K_{0}\left[R_{j} \sqrt{f_{2 j}(z)}\right] A_{j 2}+I_{0}\left[R_{j} \sqrt{f_{2 j}(z)}\right] B_{j 2}=0(26 \mathrm{c})
\end{aligned}
$$

Let:

$$
\begin{aligned}
& \psi_{m, n}(\alpha, \beta, y) \\
& =K_{m}(\alpha y) I_{n}(\beta y)+(-1)^{m-n+1} I_{m}(\alpha y) K_{n}(\beta y)
\end{aligned}
$$

Where: $m, n$ are real constant, $I_{v}(\cdot), K_{v}(\cdot)$ are first and second kind of modified Bessel function respectively.

Similar kernel function is defined as follows:

$$
\psi(r, \mathrm{z})=\frac{\left[\begin{array}{l}
\gamma_{j f} \psi_{0,0}\left(r, \beta r_{w j}, \sqrt{f_{1 j}(z)}\right) \\
+\psi^{*}\left(\beta r_{w j}, z\right) \psi_{0,1}\left(r, \beta r_{w j}, \sqrt{f_{1 j}(z)}\right)
\end{array}\right]}{\left[\begin{array}{l}
\gamma_{j f} \psi_{1,0}\left(\mathrm{r}_{w j}, \beta \mathrm{r}_{w j}, \sqrt{f_{1 j}(z)}\right) \\
+\psi^{*}\left(\beta \mathrm{r}_{w j}, z\right) \psi_{1,1}\left(\mathrm{r}_{w j}, \beta \mathrm{r}_{w j}, \sqrt{f_{1 j}(z)}\right)
\end{array}\right]}
$$

Where:

When outer boundary is infinity:

$$
\psi^{*}(r, z)=\sqrt{\frac{f_{1 j}(z)}{f_{2 j}(z)}} \frac{K_{0}\left(r \sqrt{f_{2 j}(z)}\right)}{K_{1}\left(\beta \mathrm{r}_{w j} \sqrt{f_{2 j}(z)}\right)} .
$$

When outer boundary is closed: 


$$
\psi^{*}(r, z)=\sqrt{\frac{f_{1 j}(z)}{f_{2 j}(z)}} \frac{\psi_{0,1}\left(r, R_{j}, \sqrt{f_{2 j}(z)}\right)}{\psi_{1,1}\left(\beta \mathrm{r}_{w j}, R_{j}, \sqrt{f_{2 j}(z)}\right)} .
$$

When outer boundary is constant pressure:

$$
\psi^{*}(r, z)=\sqrt{\frac{f_{1 j}(z)}{f_{2 j}(z)}} \frac{\psi_{0,0}\left(r, R_{j}, \sqrt{f_{2 j}(z)}\right)}{\psi_{1,0}\left(\beta \mathrm{r}_{w j}, R_{j}, \sqrt{f_{2 j}(z)}\right)} .
$$

$A_{j 1} 、 B_{j 1}, A_{j 2}, B_{j 2}$ are obtained by solving the equations (22-26)and using Grammer rule, therefore similar structure of the solution with reservoir pressure distribution in the Laplace space are obtained by putting the $A_{j 1}, B_{j 1}, A_{j 2}, B_{j 2}$ plug into the formula (18)-(21) and using similar kernel function.

$$
\begin{aligned}
& \bar{P}_{1 j f}=\frac{B q}{z} \cdot \frac{1}{2 \pi \sum_{j=1}^{N} \gamma_{1 j} \mathrm{r}_{w j} \sqrt{f_{1 j}(z)} \frac{1}{\left[\begin{array}{l}
\psi\left(\mathrm{r}_{w j}, z\right) \\
+S_{j} \mathrm{r}_{w j} \sqrt{f_{1 j}(z)}
\end{array}\right]}+C z} \\
& \cdot \frac{\psi(\mathrm{r}, z)}{\psi\left(\mathrm{r}_{w j}, z\right)+S_{j} \mathrm{r}_{w j} \sqrt{f_{1 j}(z)}} \\
& \bar{P}_{1 j m}=\frac{\lambda_{1 j}}{\delta_{1 j m} z+\lambda_{1 j}} \cdot \frac{B q}{z} \\
& \frac{1}{2 \pi \sum_{j=1}^{N} \gamma_{1 j} \mathrm{r}_{w j} \sqrt{f_{1 j}(z)} \frac{1}{\left[\begin{array}{l}
\psi\left(\mathrm{r}_{w j}, z\right) \\
\left.+S_{j} \mathrm{r}_{w j} \sqrt{f_{1 j}(z)}\right]
\end{array}\right]}+C z} \\
& \cdot \frac{\psi(\mathrm{r}, z)}{\psi\left(\mathrm{r}_{w j}, z\right)+S_{j} \mathrm{r}_{w j} \sqrt{f_{1 j}(z)}}
\end{aligned}
$$

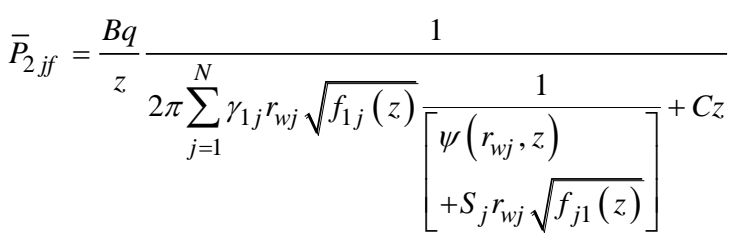

$$
\begin{aligned}
& \cdot \frac{1}{\psi\left(r_{w j}, z\right)+S_{j} r_{w j} \sqrt{f_{1 j}(z)}} \\
& \cdot \frac{\psi_{0,1}\left(\beta r_{w j}, \beta r_{w j}, \sqrt{f_{1 j}(z)}\right) \psi^{*}(r, z)}{\left[\begin{array}{l}
\gamma_{j f} \psi_{1,0}\left(r_{w j}, \beta r_{w j}, \sqrt{f_{1 j}(z)}\right) \\
+\psi^{*}\left(\beta r_{w j}, z\right) \psi_{1,1}\left(r_{w j}, \beta r_{w j}, \sqrt{f_{1 j}(z)}\right)
\end{array}\right]} \\
& \bar{P}_{2 j m}=\frac{\lambda_{2 j}}{\delta_{2 j m} z+\lambda_{2 j}} \cdot \frac{B q}{z} \\
& \frac{1}{2 \pi \sum_{j=1}^{N} \gamma_{1 j} r_{w j} \sqrt{f_{1 j}(z)} \frac{1}{\left[\begin{array}{l}
\psi\left(r_{w j}, z\right) \\
\left.+S_{j} r_{w j} \sqrt{f_{j 1}(z)}\right]
\end{array}\right]}+C z}
\end{aligned}
$$

\section{Conclusion and Cognition}

1 Observing the reservoir pressure (27) and (29); bottom hole pressure (31), we find that they have the following relation.

$$
\begin{gathered}
\bar{P}_{1 j f}=\bar{P}_{w} \cdot \frac{\psi(\mathrm{r}, z)}{\psi\left(\mathrm{r}_{w j}, z\right)+S_{j} \mathrm{r}_{w j} \sqrt{f_{1 j}(z)}} \\
\bar{P}_{2 j f}=\bar{P}_{w} \cdot \frac{1}{\psi\left(r_{w j}, z\right)+S_{j} r_{w j} \sqrt{f_{1 j}(z)}} \\
\cdot \frac{\psi_{0,1}\left(\beta r_{w j}, \beta r_{w j}, \sqrt{f_{1 j}(z)}\right) \psi^{*}(r, z)}{\left[\begin{array}{l}
\gamma_{j f} \psi_{1,0}\left(r_{w j}, \beta r_{w j}, \sqrt{f_{1 j}(z)}\right) \\
+\psi^{*}\left(\beta r_{w j}, z\right) \psi_{1,1}\left(r_{w j}, \beta r_{w j}, \sqrt{f_{1 j}(z)}\right)
\end{array}\right]} .
\end{gathered}
$$

2 With regard to the similar structure of the solution with reservoir pressure distribution in the Laplace space (27)-(30), when outer boundary condition changes, the solution with reservoir pressure distribution in the Laplace space is easily obtained by changing similar kernel function.

3 Similar construction method is a kind of primary and algebraic method, it avoids the complex derivation, it is simple and effective for solving the seepage model for the multilayer composite reservoir with the double porosity.

4 With regard to the similar structure of solution in the Laplace space, it provides immensely convenient for compiling corresponding analysis software of well test, and It also provides scientific basis for tremendous numerical simulation of oil-gas reservoir and other engineering application of seepage theory

\section{Explanation of Symbols}

$p_{o j}$ is initial pressure of each layer in the reservoir $(\mathrm{MPa}) ; r_{w}$ is well radius $(m) ; r_{w j}$ is well radius of 
the $j$ layer $(m) ; p_{j}$ is pressure of the $j$ layer at time $t$ ( $M P a) ; P_{j}$ is pressure drop of the $j$ layer $(M P a) ; S_{j}$ is skin effect of the $j$ layer(dimensionless); $C$ is coefficient of well-bore storage $\left(\mathrm{m}^{3} / \mathrm{MPa}\right) ; B$ is coefficient of crude oil volume $\left(\mathrm{m}^{3} / \mathrm{m}^{3}\right) ; k_{j}$ is permeability of the $j$ layer $\left(\mu m^{2}\right) ; C_{t j}$ is coefficient of integrated compression for the $j$ layer( $1 / M P a) ; \varphi_{j}$ is porosity of $j$ layer( $\% \quad) ; \mu_{j}$ is fluid viscosity of the $j$ layer( $M P a \cdot s \quad) ; h_{j}$ is thickness of the $j$ layer( $m) ; q_{j}$ is production of the $j$ layer $\left(m^{3} / d\right) ; q$ is production $\left(m^{3} / d\right) ; R_{j}$ is outer boundary radius of the $j$ layer $(m) ; R$ is outer boundary radius $(m) ; t$ is time $(h) ; Z$ is variable of Laplace space.

\section{Acknowledgment}

This Project is supported by the Scientific Research Fund of Sichuan Provincial Education Department of China under Grant No.12ZA164.

\section{References}

[1] Shunchu Li, Bingguang Huang. Laplace transform and Bessel functions and the theoretical basis of well test analysis. Beijing: Petroleum Industry Press, 2000.

[2] Kuchuk F J, Ayestaran L et al. Application of Multilayer Testing and Analysis: A Field Case. Paper SPE15419 presented at 61 Annual Technical Conference and Exhibition in New Orieans,LA October 5-8,1986.

[3] Xianzhi Xu, Guohua Kuang, Fenglei Chen, etc. Method of well test for multilayer commingled producing (in Chinese). Acta Petrolei Sinica,1999,20(5):43-47.

[4] Shunchu Li, Puzai Zhang, Bingguang Huang, etc. General solution of the pressure distribution in multilayer reservoir (in Chinese). Journal of Southwest Petroleum Institute, 2002, 24(4):28-29.

[5] Liehui Zhang, Haitao Wang, Yonglu Jia,etc. Model of two-layered reservoir with crossflow and dual porosity media (in Chinese). Journal of Southwest Petroleum Institute, 2009, 31(5):178--184.

[6] Jingjing Guo, Liehui Zhang, Haitao Wang. Productivity analysis on commingled production wells in layered dual porosity reservoirs(in Chinese). Journal of Hydrodynamics, 2011, 26(6):704-712.

[7] Quanyong Li, Shunchu Li, Wei Li, etc. Solution to Base on the Similar Structure of the Double Porosity-Multilayer Reservoir(in Chinese). College Mathematics, 2013, 29(1): 56-60.

[8] Shunchu Li, Minhui Jia. The formal similarity of solutions on the class of differential equation(in Chinese). Journal of UEST of China, 2004, 33(Supp.):95-98.

[9] Lijie Guo, Shunchu Li, Cuicui Sheng. Similar structure of solutions for radial flow of fractal reservoir(in Chinese). FaultBlock Oil \& Gas Field, 2012, 19(1): 114-116.

[10] Shunchu Li, Junchao Wang, Li Xu. The Similar Structure of Solutions to the Spherical Fluids Flow Problems for a Composite Reservoir(in Chinese). Journal of Mathematics in Practice and Theory, 2014,44(3):122-127.

[11] Liya Chen, Shunchu Li, Xia Lai.Solution analysis of bottom hole pressure distribution in the dual porosity composite reservoir(in Chinese). Drilling \& Production Technology, 2010, 33(5): 5254(to61).

[12] Ying Chi, Shunchu Li, Quanyong Li. Nonlinear flow solution of fractal composite reservoir model based on similar structure(in Chinese). Drilling \& Production Technology, 2011, 34(2):4648(to108).

[13] Junchao Wang, Shunchu Li, Li Xu. Spherical fluids flow models in composite reservoir based on similar structure of solution (in Chinese). Journal of Guilin University of Technology, 2012, 32(4): 624-627.

[14] Xuxia Xiao, Shunchu Li. The similar structure of solutions for the boundary value problem of Euler' $s$ hypergeometric differential equation(in Chinese). Journal of Inner Mongolia Normal University (Natural Science Edition), 2012, 41(6): 597-600(-603).

[15] Furong Wang, Shunchu Li, Dongxu $\mathrm{Xu}$. The similarity construction method of a kind of boundary value problem of airy equation(in Chinese). Journal of Hubei Normal University (Natural Science), 2013, 33(1):79-85.

[16] Cuicui Sheng, Jinzhou Zhao, Yong-Ming Li, Shunchu Li, Hu Jia. Similar construction method of solution for solving the mathematical model of fractal reservoir with spherical flow. Journal of Applied Mathematics, vol. 2013, Article ID 219218, 8 pages, 2013.

[17] Yong Wang, Xitao Bao, Shunchu Li. Similar constructive method for solving a non-linearly spherical percolation model. Telkomnika Indonesian Journal of Electrical Engineering, Vol. 11, No.3, pp.1393-1402, 2013.

[18] Li Xu, Xiangjun Liu, Lixi Liang, Shunchu Li, and Longtao Zhou. The similar structure method for solving the model of fractal dualporosity reservoir. Mathematical Problems in Engineering, Volume 2013, Article ID 954106, 9 pages.

[19] Pengshe Zheng, Shunchu Li, Lihui Leng, Dongdong Gui. Similar construction method of boundary value problems of a nonlinear composite modified bessel equations(in Chinese). Journal of Xinyang Normal University (Natural Science Edition), 2014, Vol.27 (4): 490-492(-504).

[20] Kamke E. The manual of Ordinary differential equation. Beijing: Science Press, 1977. 